\title{
Influence of Culture, Transparency, Trust, and Degree of Automation on Automation Use
}

\author{
Shih-Yi Chien, Member, IEEE, Michael Lewis, Member, IEEE, Katia Sycara, Fellow, IEEE, \\ Asiye Kumru, Jyi-Shane Liu
}

\begin{abstract}
The reported study compares groups of 120 participants each, from the United States, Taiwan, and Turkey interacting with versions of an automated path planner that vary in transparency and degree of automation. The nationalities were selected in accordance with the theory of Cultural Syndromes as representatives of Dignity (US), Face (Taiwan), and Honor (Turkey) cultures and were predicted to differ in readiness to trust automation, degree of transparency required to use automation, and willingness to use systems with high degrees of automation. Three experimental conditions were tested. In the first, highlight, path conflicts were highlighted leaving rerouting to the participant. In the second, re-planner made requests for permission to reroute when a path conflict was detected. The third combined condition increased transparency of the re-planner by combining highlighting with rerouting to make the conflict on which decision was based visible to the user. A novel framework relating transparency, stages of automation, and trust in automation is proposed in which transparency plays a primary role in decisions to use automation but is supplemented by trust where there is insufficient information otherwise. Hypothesized cultural effects and framework predictions were confirmed.
\end{abstract}

Index Terms - cultural differences, degree of automation, automation transparency, trust in automation

\section{INTRODUCTION}

A S the global spread of technological innovation increases, it is necessary to study the factors that determine the ways in which users in different cultures adopt and use technology. It is particularly important that products such as advanced automation designed by and for use within one country and culture be also usable in other countries and cultures. This

This paper was submitted for review on May 31, 2018. This work was supported in part by the Air Force Office of Scientific Research under Grant FA9550-13-1-0129

Shih-Yi Chien is with National Chengchi University, Taipei, Taiwan (email: gsechien@gmail.com)

Michael Lewis is with the University of Pittsburgh, Pittsburgh, PA (e-mail: ml@sis.pitt.edu)

Katia Sycara is with Carnegie Mellon University, Pittsburgh, PA (e-mail: katia@cs.cmu.edu

Asiye Kumru is with Özyeğin University, Istanbul, Turkey (e-mail: asiye.kumru@ozyegin.edu.tr

Jyi-Shane Liu is with National Chengchi University, Taipei, Taiwan (e-mail: jyishane.liu@gmail.com) challenge involves many different facets of automation and in particular factors that affect the choice to use automation. This study investigates the influences of transparency and degree of automation as mediated by culture on the decision to use automation. Section II introduces the theory of Cultural Syndromes, represented in this study by U.S., Taiwan, and Turkey, and advances hypotheses about the role these syndromes might play in the decision to use automation. Section III presents a unifying framework for predicting the effects of transparency, trust, degree of automation (DOA), on one another and the decision to use automation. Section IV presents the simulation environment and experimental design. Results are presented in Section $\mathrm{V}$ followed by discussion in Section VI and conclusion in Section VII.

\section{CULTURE AND AUTOMATION}

Culture has been defined as the unique nature of a social group with regards to values, beliefs, norms, and practices [1]. Cultures can have a central theme or syndrome, which is a compilation of shared beliefs and practices. The majority of cross-cultural research has relied on the cultural themes of individualism and collectivism as well as Hofstede's cultural dimensions [2] which focus on values. While these constructs are useful for predicting and explaining attitudes and social organization they do not directly predict behavior. Recent research focusing on norms (typical patterns of behavior) found in Honor, Face, and Dignity cultures provides a more direct and predictive framework for how people interact, form relationships and handle conflicts [3]. To study cross cultural effects of trust in automation we have collected data from three countries, United States (US), Taiwan (TW), and Turkey (TK), representative of the three cultural syndromes and varying significantly from one another on Hofstede's dimensions of power distance, uncertainty avoidance, and individualism as measured by CVScale [4]. Because no validated measure of individual adherence to cultural syndromes was available at the time of this study our comparisons are based on intact groups and cannot exclude differences between college populations in the U.S., Taiwan, and Turkey unrelated to their national cultures. These differences could include possible differences in computer skills and experience with automated systems or 
differences between academic disciplines Information Science (US) and Business (TW, TK).

\section{A. Cultural Syndromes}

Cultural syndromes encompass cultures of Dignity, Honor, and Face, which contrast with respect to the meaning and importance that are given to norms of exchange, reciprocity, punishment, honesty, and trustworthiness.

In Dignity cultures, prevalent in Western Europe and North America, one's self-worth is derived internally. It is only evaluated by the individual's own standards [5]. Dignity cultures are high on individualism and low on power distance. The context that surrounds interactions is egalitarian, consisting of autonomous individuals who focus on personal, individual goals, supported by an effective system of law that enforces contracts and rights [5]. In these cultures, people generally have a "swift trust" assumption: others deserve to be trusted until they prove otherwise. These characteristics would lead to the belief that operators from Dignity cultures will be quick to trust in automation.

Face cultures are prevalent in East Asian societies where one's self-worth is derived externally. Self-worth is the view that others have of the individual and is based on social interactions with others. It is stable so long as the social hierarchy in which the person interacts is stable [5]. So, selfworth is interdependent with a person's role in a stable social hierarchy, and on fulfillment of role obligations. In these cultures, people can lose face if others disapprove of their actions and behaviors [5]. Face cultures are high in collectivism and high in power distance. People interact in stable hierarchies, and social interactions are governed by norms imposed by social institutions, such as religion, family, community or the state. People's conformity to those norms is monitored and if necessary, managed by institutional sanctioning. Because of this institutional monitoring and sanctioning, people can engage in smooth social interactions in the absence of trust [6].

Honor Cultures can be found in the Middle East, Latin America, and Mediterranean countries. People's self-worth is dependent on interactions with others and one's perception of self. Honor cultures are in the middle range on Hofstede's dimensions of collectivism and power distance. In these cultures, honor, linked to self-worth, must be claimed as well as paid to others [5]. Honor cultures manifest with a reputation for toughness in protecting the self and family and involve not letting others take advantage of you [7]. The social context of Honor cultures is unstable social hierarchies. Consequently, members of an Honor culture tend to have slow trust (low trust at the beginning of interactions) and low trust in laws and institutions.

Our hypotheses derived from this theory are:

H1: Individuals from dignity cultures (e.g., United States) will have a higher level of dispositional trust due to safety guarantees afforded by rule of law, whereas the individuals from an honor culture (e.g., Turkey) will have a lower level of dispositional trust due to norms promoting self-protection.

H2: Honor culture operators will be less likely to comply with high degree of automation (DOA) or low transparency automation than Face or Dignity culture operators and will require greater support than Dignity and Face cultures because of their self-protective stance.

H3: Lack of transparency will lower ratings of trust of operators from all cultures due to weakening of evidence about system operation and performance.

H4: Face culture (e.g., Taiwan) operators will exhibit automation bias by accepting recommendations from automation even if their basis is not well understood due to their authoritative source, while Dignity and Honor culture operators will be less likely to trust or accept recommendations on this basis.

\section{TRANSPARENCY, TRUST, AND DEGREE OF AUTOMATION}

Beck et al. [8] have termed the choice to use and the manner in which automation is used the automation usage decision. An operator is presumed to choose to depend on automation under circumstances in which she expects to benefit from use and reject it otherwise. Automation transparency refers to the extent to which automation provides the information needed for a human to make accurate predictions of its behavior in order to make this decision. In conventional closed loop control, such as a household thermostat (Fig.1), the state of the system (temperature) is sensed, this data is processed (compared to temperature setting) and a response is generated (furnace turned on or off). Conventional thermostats make themselves transparent by displaying information from all three of these stages: sensed and reference temperatures and indication of furnace operation. One might still use a less transparent, opaque thermostat that could only be turned on or off but predicting its behavior would now require beliefs about current temperature, temperature setting, and how the thermostat operates. Finally, our thermostat could be a less than fully automated manual thermostat providing a display for the user to compare current and reference temperatures but requiring the user to press a button to start the furnace.

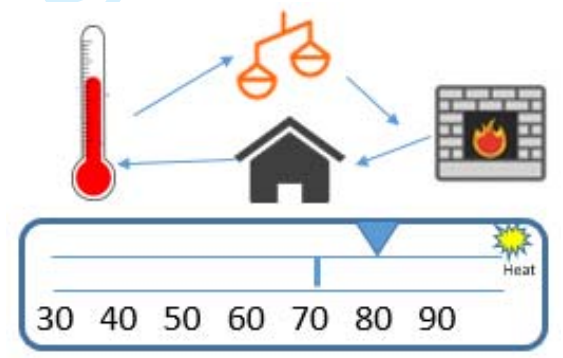

Fig. 1-Diagram shows control loop. Thermometer measures temperature in house which is compared with temperature setting (scales). If measured temperature is less than setting, furnace is turned on and heat indication on thermostat illuminated; if below they are turned off. The furnace affects the temperature within the house completing the loop. For the conventional thermostat the user can see all parts of the control loop. For the manual thermostat the user can see the temperature and setting but must make the comparison and turn the furnace on/off herself. The opaque thermostat shows nothing. The user must judge by the comfort of the house whether thermostat is functioning properly.

In this paper we investigate the relations between transparency, trust, degree of automation and dependence on 
automation. Because these constructs have historically been treated as distinct topics we will review each in turn.

\section{A. Trust in Automation}

Trust in automation is an attitude, which can act as an intervening variable to mediate a human's decision to use or not use available automation [9]-[11]. Increasing the appropriateness of dependence on automation is presumed to depend on the calibration of this trust [12]. Trust, however, is not an exclusive determinant of usage, with other factors such as task load [9], [13] frequently found to affect usage independent of trust. Even when trust and dependence on automation are affected by the same factors, mediation may be absent as in the case of alarms [15] for which rates of agreement can be predicted from probability matching without reference to trust. We hypothesize that trust will become a determinant of dependence primarily in situations where information for predicting automation behavior is incomplete.

Accumulated research suggests that trust in automation is affected by system characteristics such as reliability degree of automation [16], [17], and transparency [10], [18], while individual characteristics such as self confidence in performing a task [19] culture [20], and environmental characteristics such as task load [21], [22] and risk [22] may affect either trust, dependence, or both.

\section{B. Degree of Automation}

The effects of automation on usage and trust depend jointly on what aspects of a task are automated and how they are automated. These distinctions have commonly been organized as levels, stages, or degrees of automation. The oldest of these, Levels of Automation (LOA) [19], focuses on locus of control (human 1-5, or automation 7-10) and proceeds from aspects of the task involving information to those dealing with actions. A revision of this taxonomy, Stages of Automation [20], emphasized the progression from information to action by postulating four stages of automation: information acquisition, information analysis, decision selection, and action implementation. Guidance based on this model [12], [23], [24], later termed degree of automation (DOA), favors higher degrees of automation at early stages and lower degrees of automation at later ones. Fig. 2 shows automation profiles for transparency of these stages [23] for the thermostats in Fig. 1 illustrating the "end-to-end" principle. The conventional thermostat, A, with transparency at each of the involved stages is fully transparent making its behavior completely predictable (furnace off above setpoint and on below setpoint). The manual thermostat, B, is also fully transparent through the information analysis stage where it ends because user can see both the current temperature and the setpoint providing all the information needed for the choice to turn the furnace on or off. For the opaque thermostat, $\mathrm{C}$, automation behavior cannot be predicted directly because the stages preceding the action are unobservable. Instead, the decision to use or not use the opaque thermostat must rely on beliefs about what it will do, i.e. trust. Because later stages of automation often presuppose automation at earlier stages without requiring them to be observed, later stage automation is typically more opaque as pointed out by [24] and illustrated in our thermostat example. Earlier stages are likewise more likely to be fully transparent because there are few or no prior stages to be made transparent.

For later stage automation the operator may lack the ability to perform the task independently, as well, and thus be less able to predict or evaluate the system's performance [24]. Research on the effects of automation failures finds a similar tradeoff with operator workload lowered by automation but situation

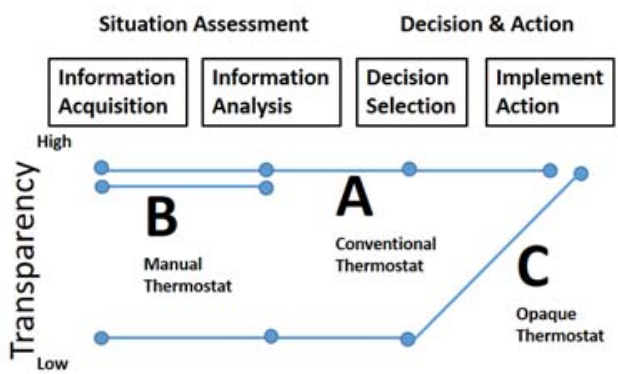

Fig. 2. Transparency profiles based on Parasuraman, Wickens, and Sheridan (2000) automation stages-conventional thermostat A is observable at each stage of automation, manual thermostat $\mathrm{B}$ is observable at the two stages it includes, opaque thermostat $\mathrm{C}$ is only observable at the fourth stage through furnace being on or off.

awareness (SA) and recovery from failures significantly degraded by high degrees of automation. This leads to recommendations for choosing medium levels of automation [25], [26] in order to benefit from lowered workload while maintaining sufficient SA to manage failures.

A smaller number of studies have measured the effects of DOA on trust. [16] found ratings of trust higher in a condition prioritizing a list of possible engagements than in either an unprioritized list or higher levels of automation. [27] similarly found a lower level of automation preferred for aiding an air traffic control (ATC) task. [26] report higher ratings of trust for management by consent (lower LOA) than for management by exception (higher LOA) as well. A related finding by [17] showed that making results of automation at the information analysis stage observable in addition to an automated action served to increase trust. While limited, these results suggest that the effects of DOA on trust parallel those on performance with lower levels/earlier stages of automation inducing greater trust and higher usage than higher DOAs

\section{Transparency}

Transparency can occur at any stage of automation but may be most effective when preceding stages are transparent as well. Despite this, many transparency manipulations have targeted performance at the execution stage alone. Attributes such as system reliability are often assumed to be directly observable to users yet may be perceived inaccurately. [21], for example, found trust and dependence to be influenced by cover stories. Other studies [8] have found supplying knowledge of results to lead to better trust calibration and improved dependence. Annotating decisions with confidence judgments is another widely used technique for providing greater insight into system performance. This has been done variously by providing probabilities of success [10], [28], confidence in detection [28] 
or confidence (Q values) in selected robot actions [11].

Transparency at the situation assessment stage provides teleological (why) explanations which may be incomplete, simply conveying relevant features contributing to an automation decision [29] or supplying the logic behind the decision [30] as well. Teleological explanations are both preferred by humans [31] and conform to our premise that endto-end transparency will lead to fuller prediction and more accurate decisions. Transparency of purpose, by contrast, is usually informally conveyed through instructions or the demand characteristics of an experiment, however, when explicitly manipulated in [17], effects on trust and dependence were found.

\section{Synthesizing Trust, DOA, and Transparency}

Trust, DOA, and transparency can be related in a unified framework in which use of automation is treated as a rational decision based on a human's expectation of benefiting from the automation's predicted behavior. When there is no uncertainty (automation is fully transparent) this decision can be based entirely on observation. Where uncertainty exists trust contributes to the automation usage decision. Automation transparency can affect the decision to use both directly and indirectly through its effects on learned trust.

The experiment compares experimental conditions that:

1. automate and display information from the information analysis stage (full transparency)-HL highlight condition in experiment

2. automate the action stage without information from the earlier stages on which it is based (opaque)- PR path-replan condition in experiment

3. display information from information analysis stage and automate action (full transparency). PRHLpath-replan and highlight condition from experiment.

We hypothesize that:

TH 1 trust will be rated higher for the transparent conditions and participants will comply with the transparent response of condition 3 at a higher rate than the opaque one of condition 2.

TH 2 In the presence of uncertainty about automation behavior in condition 2, we hypothesize that trust will play a role in automation dependence.

TH 3 For transparent condition-3 we hypothesize a lessened role for trust than condition-2 along with a higher rate of dependence.

\section{EXPERIMENT}

This paper reports on the navigation task from a dual task study conducted in the U.S., Taiwan, and Turkey. The study was conducted in the context of a larger research project in which a cross-cultural measure of trust was developed. Data from this study provided both a basis for investigating crosscultural effects of trust in automation and for convergent and predictive validation of the trust measure.

The experimental simulation was developed by modifying an existing multi-UAV simulation, RESCHU [32] (see Fig. 3), by adding a likelihood display for the payload task and adding
Dubins path dynamics to the navigation task. Five UAVs were routed between sequences of potential targets. Operators performed two tasks: identifying and attacking hostile targets (payload task), and rerouting the UAVs' paths to avoid conflicts (navigation task). RESCHU provided a payload window (shown at the top left in Fig. 3) for target detection tasks and a map display (shown in the right window in Fig. 3) for UAV navigation tasks.

An autonomous path planner was used to generate visible UAV paths following a shortest-distance criterion. Because UAVs were independently assigned to new targets, conflicting paths risking collision could arise. In addition, the navigation task required monitoring UAV paths to avoid passing through hazard areas (shown as yellow regions in Fig. 4b) which appeared at random intervals and locations. When a UAV confronts a risk, such as conflicting paths or entering a hazard area, the operator must reroute the UAV by adding waypoints to avoid collisions and maneuver around threats. To add realistic complexity to rerouting, UAVs diverted by the path planner followed Dubins paths [33] which enforce a turning radius similar to the behavior of fixed wing air craft. A Dubins path is the shortest path that connects two points in the Euclidean plane with a constraint on curvature and results in path segments that are either of maximum curvature or straight. Such irregular paths are more difficult for users to understand and predict strengthening the roles trust and transparency might play in their dependence decisions.

\section{A. Automated Assistance}

A conflict detector predicted conflicts between UAV paths or paths and hazards when the area of risk came within a prescribed range of a UAV. To aid the operator this area was highlighted to indicate the risk, a path which would avoid the risk was generated, or both were provided. The conflict detector was $90 \%$ reliable generating erroneous alerts, $10 \%$ of the time, while the path planner was completely reliable. This equated the automation conditions for reliability, as errors in all three arose solely from the conflict detector. Vehicle to Vehicle (V-V) and Vehicle to Hazard (V-H) damages were assessed as a sum of the durations a vehicle spent in dangerous proximity to other vehicles or within a hazard region.

To alert the operator to an impending risk detected by the conflict detector, the highlight aid placed a red square on the map (Fig. 4a) in the region in which minimum separation was predicted to be violated or added red dots (Fig. 4b) at the points at which the UAV was expected to enter and exit a hazard area. The operator then needed to manually add waypoints to divert the UAV around the risk. In automated response conditions path planning was initiated when a risk was detected by the conflict detector. The Path Planner generated an additional waypoint defining a path that would avoid the risk and undimmed the UAV's 'AUTO' button making it available for use. The user could then either accept the unseen path by clicking 'AUTO', supply a waypoint manually, or do nothing (Fig. 5). If the new plan is selected the UAV will avoid the risk but incur additional costs in traveling distance and time providing an incentive for rejecting false alarms. In the 


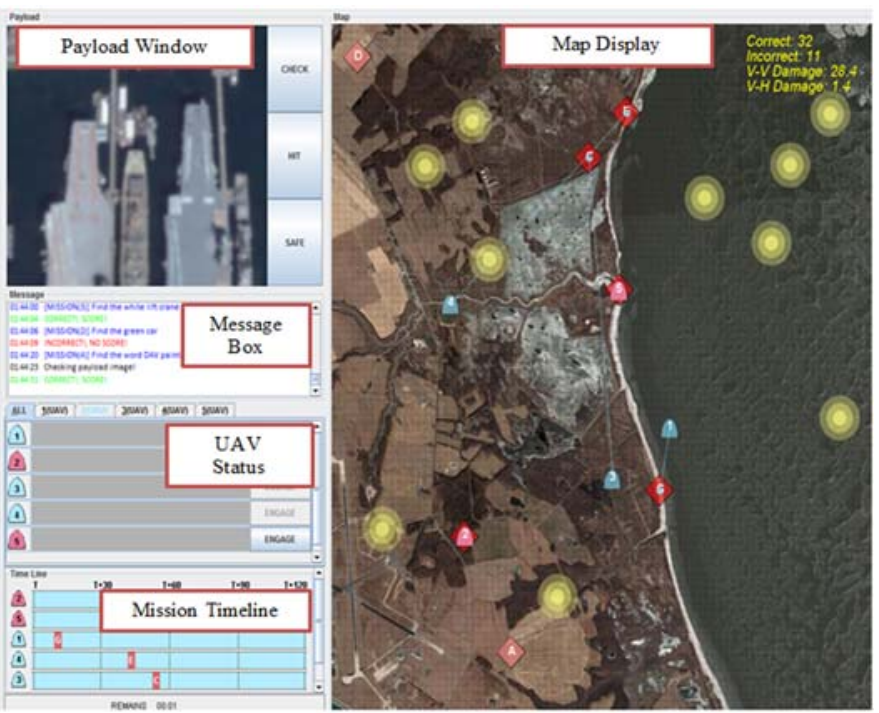

Fig. 3. The RESCHU user interface. The map window shows the numbered UAVs (blue ovals) with paths to targets (red diamonds); threat areas are marked by the yellow circles.
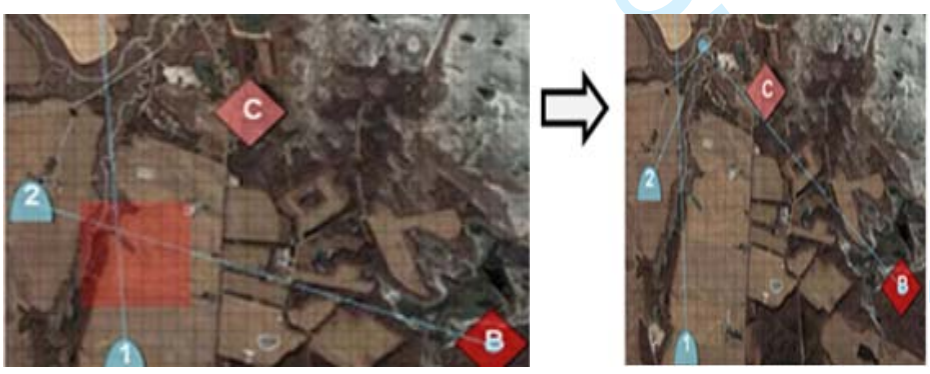

(4a) Adding waypoints to avoid path conflicts
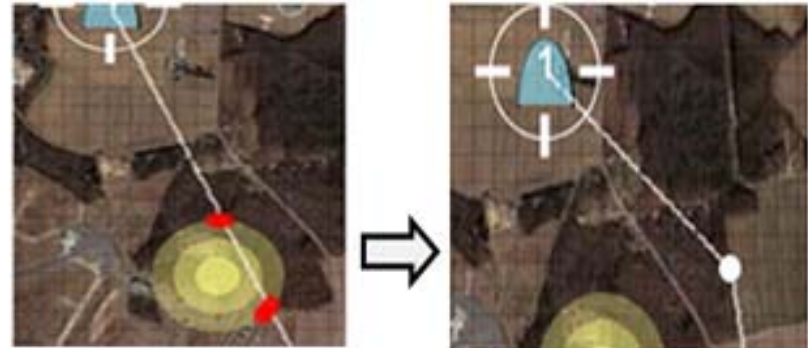

(4b) Adding waypoints to avoid threat areas Fig. 4. Adding UAV's waypoints to avoid collisions.
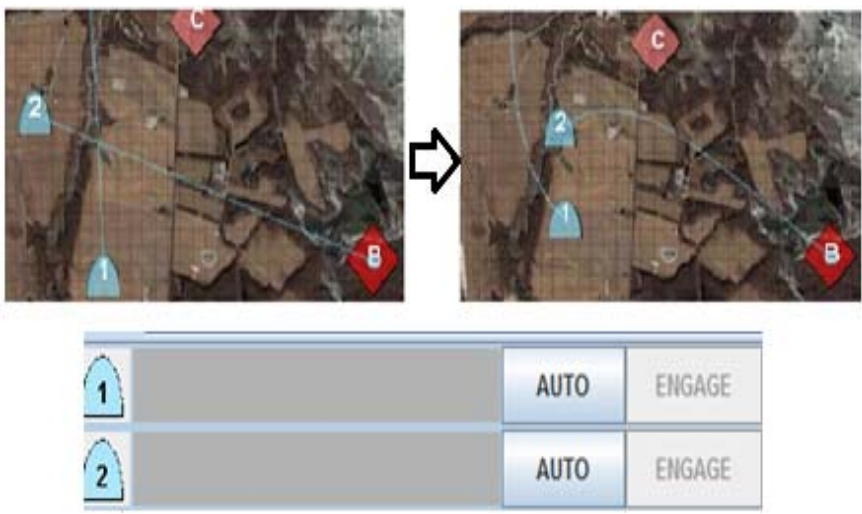

Fig. 5. By clicking the auto button, new paths are applied to divert the UAVs from the collision course.

Highlight + Planner condition both highlighting of risks and the option of selecting an automated plan are available.

\section{B. Universal Trust in Automation Measure}

As part of this research we have developed a measure of trust in automation, Universal Trust in Automation (UTA), that has been validated across large samples in three diverse cultures: United States, Taiwan, and Turkey representatives of dignity, face, and honor cultures respectively [5]. Following standard test development methods, a psychometric measure with three dominant dimensions was developed. Upon examination of items these dimensions were found to correspond roughly to the performance, purpose, and process dimensions hypothesized by [12]. One distinct 9 item scale corresponds to the dispositional and situational sources of variation hypothesized by [9] while the second 9 item scale measures trust as learned through experience with the system. Scales, data, and analyses are archived at OpenICPSR [34]. Reported full scale scores are an average of their dimensional subscales.

\section{Experimental Conditions}

Degree of automation (DOA), trust, and transparency are major factors known to affect the decision to use automation. To investigate these effects, the present experiment manipulated reliability of the payload task (reported in [35]) and taskload and DOA/transparency of the (reported here) navigation task. The tasks can safely be analyzed independently because all interactions between the payload task (two levels of reliability) with navigation measures: trust in Conflict Detector $(\mathrm{F} 2,504=.572, \mathrm{p}=.565, \eta 2=.002)$, vehicleto-vehicle damages $(\mathrm{F} 3,672=.222, \mathrm{p}=.881, \eta 2=.001)$, vehicleto-hazard damages $(\mathrm{F} 3,672=.516, \mathrm{p}=.672, \eta 2=.002)$, and compliance $(\mathrm{F} 1,336=.768, \mathrm{p}=.382, \eta 2=.002)$ were found nonsignificant.

DOA and transparency were manipulated in four experimental conditions: a monitoring control condition without conflict detection (Cntl) and three aided conditions replicating the automation profiles of the thermostats in the introduction: Highlight (HL) in which potential V-V and V-H conflicts were highlighted (manual thermostat), Path Replanner (PR) in which rerouting waypoints were proposed to avoid vehicle to vehicle conflicts (opaque thermostat), and Highlight + Planner (PRHL) in which conflict detection led to both highlighting and a rerouting proposal (conventional thermostat). Data were collected from the U.S., Taiwan, and Turkey, countries selected to provide representatives of the three cultural syndromes, US- Dignity, Taiwan-Face and Turkey-Honor. The navigation task followed a mixed repeatedmeasures design, with countries (U.S., Taiwan, and Turkey) and information transparency/DOA (control, PR, HL, PRHL) as the between-subject factors; and counterbalanced taskload as the within-participant variable. Task load was manipulated through changes to the UAVs' moving speed, in which the vehicles moved at 5.0 pixels/second in the high taskload (HW) condition and 2.5 pixels/second in the low taskload (LW) condition.

\section{Participants and Procedure}

120 student participants were recruited from each of the three 
countries. American participants were recruited from the University of Pittsburgh ( 80 females and 40 males with average age of 19.57, $\mathrm{SD}=2.37$ ), Taiwanese participants were recruited from National Chengchi University (80 females and 40 males with average age of 21.60, $\mathrm{SD}=2.49$ ), and Turkish participants were recruited from Özyeğin University (95 females and 25 males with average age of $21.58, \mathrm{SD}=1.63$ ). None had prior experience with air traffic control although most were frequent computer users, where in a typical week American participants spend $25.25(\mathrm{SD}=12.19)$ hours, Taiwanese participants spend $30.50(\mathrm{SD}=14.72)$ hours, and Turkish participants spend 17.67 $(\mathrm{SD}=11.50)$ hours using a computer.

After providing demographic data, participants were asked to rate their trust in automation using the dispositional scale of the UTA trust instrument. Chinese and Turkish translations of the instruments were used in their respective countries. Participants were randomly assigned to a display condition and received a 20-minute interactive training tutorial. Participants were informed that conflict detection was highly (but not perfectly) reliable. Their goal was to avoid UAV path conflicts and threat areas by adding or moving waypoints using a mouse (navigation task), while identifying and attacking as many enemy targets as possible (payload task). After training, participants began the first 10-minute experimental session followed by completing the NASA-TLX [36] and the UTA trust questionnaire [34] After a brief break, the other taskload condition was run accompanied by workload and trust measures.

\section{RESULTS}

Data were analyzed using a mixed-model ANOVA with DOA/transparency (control, PR, HL, PRHL) and countries (U.S., Taiwan, and Turkey) as the between-subject factors, and taskload (high vs. low) as the within-subject variable.

\section{A. Dispositional Trust}

All three countries differed in their predisposition to trust $\left(\mathrm{F}_{2,357}=16.225, p<.001\right)$. T-tests revealed significant differences between the U.S. and Turkey $(p<.001)$, U.S. and Taiwan $(\mathrm{p}=.007)$, and Taiwan and Turkey $(p=.003)$. The U.S. participants had the highest score in dispositional trust and the Turkish participants had the lowest, with the Taiwanese rates falling in between (Fig. 6).

The analyses also revealed significant cultural effects on subscales for performance $\left(\mathrm{F}_{2,357}=2.969, p=.053\right)$, process $\left(\mathrm{F}_{2,357}=66.225, p<.001\right)$, and task context $\left(\mathrm{F}_{2,357}=18.697, \mathrm{p}<\right.$ $.001)$. Taiwanese rates were higher than Turkey and marginally

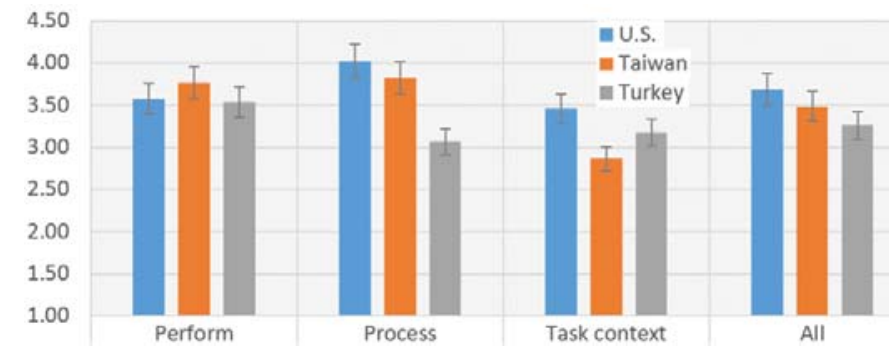

Fig. 6. Dispositional trust in automation among three cultures. higher than US in performance (TW $>\mathrm{US}, \mathrm{p}=.066$; TW $>\mathrm{TK}, p$ $=.022)-$ but lowest for task context (US>TW, $p<.001$; $\mathrm{TK}>\mathrm{TW}, p=.002$ ).

\section{B. Learned Trust}

An ANOVA revealed a main effect for country $\left(\mathrm{F}_{2,522}=12.986, p<.001\right)$ and DOA/transparency $\left(\mathrm{F}_{2,522}=18.100\right.$, $p<.001)$ on trust; however, no statistical difference was observed between taskload conditions. The U.S. and Taiwanese participants reached similar overall trust ratings, and were significantly higher than Turkish participants (US>TK, $p=$ .001 ; TW $>$ TK, $p<.001)$.

The results (Table I) also found a main effect for DOA/transparency in the overall trust ratings $\left(\mathrm{F}_{1,708}=18.221, p\right.$ $<.001)$ as well as in performance $\left(\mathrm{F}_{1,708}=14.018, p<.001\right)$, process $\left(\mathrm{F}_{1,708}=8.674, p<.001\right)$, and purpose $\left(\mathrm{F}_{1,708}=20.415, p\right.$ $<.001)$ constructs. The highest trust was observed in the HL condition and the lowest score was found in the PR condition, with PRHL in the middle. T-tests showed that both HL and PRHL were significantly higher than the PR condition across all the comparisons, confirming $\mathrm{TH}$ 1, trust will be rated higher in transparent conditions. Although little difference was

TABLE I

TRUST IN DOA/TRANSPARENCY CONDITIONS

\begin{tabular}{|c|c|c|c|}
\hline Measures & $F_{1,708}$ & $p$-valu & e Post hoc \\
\hline Performance & 13.695 & $9<.001$ & $\begin{array}{l}\mathrm{HL}>\mathrm{PR}(p<.001) \\
\mathrm{PRHL}>\mathrm{PR}(p<.001) \\
\mathrm{HL} \approx \mathrm{PRHL}(p=.318)\end{array}$ \\
\hline Process & 8.759 & $<.001$ & $\begin{array}{l}\text { HL }>\text { PR }(p<.001) \\
\text { PRHL }>\text { PR }(p=.002) \\
\text { HL } \approx \text { PRHL }(p=.336)\end{array}$ \\
\hline Purpose & 20.43 & $1<.001$ & $\begin{array}{l}\text { HL }>\text { PR }(p<.001) \\
\text { PRHL }>\text { PR }(p=.001) \\
\text { HL }>\text { PRHL }(p=.002)\end{array}$ \\
\hline $\begin{array}{l}\text { Overall } \\
\text { (average value) }\end{array}$ & 18.100 & $0<.001$ & $\begin{array}{l}\mathrm{HL}>\mathrm{PR}(p<.001) \\
\mathrm{PRHL}>\mathrm{PR}(p<.001) \\
\boldsymbol{H L}>\boldsymbol{P R H L}(\boldsymbol{p}=\mathbf{. 0 4 9})\end{array}$ \\
\hline
\end{tabular}

observed between the HL and PRHL conditions in performance and process constructs, the effects were especially prominent in the purpose construct (HL $>$ PRHL, $p=.002$ ), which resulted in significant differences in the overall comparison between the HL and PRHL aids $(p=.049)$.

\section{Transparency and Culture}

Further analysis revealed significant cultural differences between the U.S. and Turkey as well as Taiwan and Turkey in both the PR ( $p=.023$ and $p=.001$ respectively) and PRHL conditions $(p=.001$ and $p<.001$ respectively). While ratings of trust were consistently higher in the U.S. and Taiwanese population than the Turkish group, no statistical difference was found in the HL condition among the three cultural groups. US and Taiwanese participants reported similar levels of trust in HL and PRHL conditions that were considerably higher than those of Turkish participants (HL $\mathrm{p}<.02$, PRHL $\mathrm{p}<.001$ ). Notably, Turkish ratings for trust in PRHL where the system offered to replan as well as highlight the conflict were 
significantly lower $(\mathrm{p}=.001)$ than in the HL condition. While all groups expressed greater trust in the highlight conditions than in the planner alone $(p<.001)$, Turkish participants declined to trust the planner even when its actions could be verified through highlighting (PRHL).

\section{Vehicle-Vehicle and Vehicle-Hazard Damages}

Preventing UAV path conflicts and avoiding threat areas to prevent damage were primary objectives of the navigation task. The ANOVA for vehicle-vehicle (V-V) damage (i.e., path conflicts), shown in Fig. 7, found a main effect for taskload $\left(\mathrm{F}_{1,696}=8.367, p=.004\right), \mathrm{DOA} /$ transparency $\left(\mathrm{F}_{3,696}=16.077, p<\right.$ $.001)$, cultural variables $\left(\mathrm{F}_{2,696}=13.251, p<.001\right)$, and an interaction between taskload and DOA/transparency $\left(\mathrm{F}_{3,696}=3.244, p=.022\right)$. The results showed that increasing task load led to higher levels of $\mathrm{V}-\mathrm{V}$ damage. The lowest $\mathrm{V}-\mathrm{V}$ damage was found in the PRHL condition and the highest was found in the control monitoring condition, with no difference between the PR and the HL groups. Post hoc analysis found higher levels of $\mathrm{V}-\mathrm{V}$ damage for Turkish participants than American $(p<.001)$ or Taiwanese participants $(p<.001)$, with no difference found between American and Taiwanese participants.

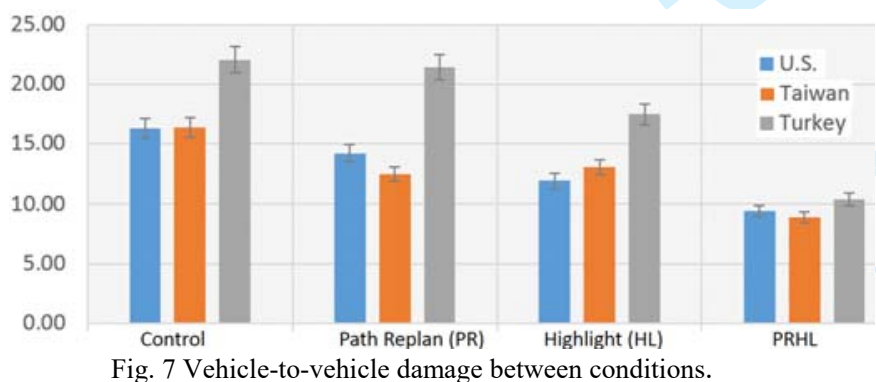

To better measure the effects of cultural factors, data were compared for country and condition. While no difference was observed for the PRHL condition, the analysis showed significant cultural differences for control (monitoring) $\left(\mathrm{F}_{2,177}=3.031, p=.05\right), \mathrm{PR}\left(\mathrm{F}_{2,177}=6.860, p=.001\right)$, and $\mathrm{HL}$ $\left(\mathrm{F}_{2,177}=4.821, p=.009\right)$ conditions, in which significantly higher damage was found for Turkish operators than American or Taiwanese participants.

The related measure of vehicle-hazard (V-H) damage (i.e., threat areas), shown in Fig. 8, revealed significant differences in taskload $\left(\mathrm{F}_{1,696}=21.371, p<.001\right)$, DOA/transparency $\left(\mathrm{F}_{3,696}=75.721, p<.001\right)$, and country $\left(\mathrm{F}_{2,696}=5.770, p=.003\right)$. Significant differences were also found for the interaction between taskload and DOA/transparency $\left(\mathrm{F}_{3,696}=196.673, p<\right.$ .001 ), Fig. 8. Higher V-H damage was found in LW than HW conditions. The lowest $\mathrm{V}-\mathrm{H}$ damage was found in the control condition while the highest damages were found in the $\mathrm{HL}$ condition, with higher damage in the PRHL condition than in the PR condition. Further analysis showed that cultural effects on $\mathrm{V}-\mathrm{H}$ damage were only observed for the PR condition between American and Turkish participants $(p=.05$ ), while the rest of the comparisons remained nonsignificant.

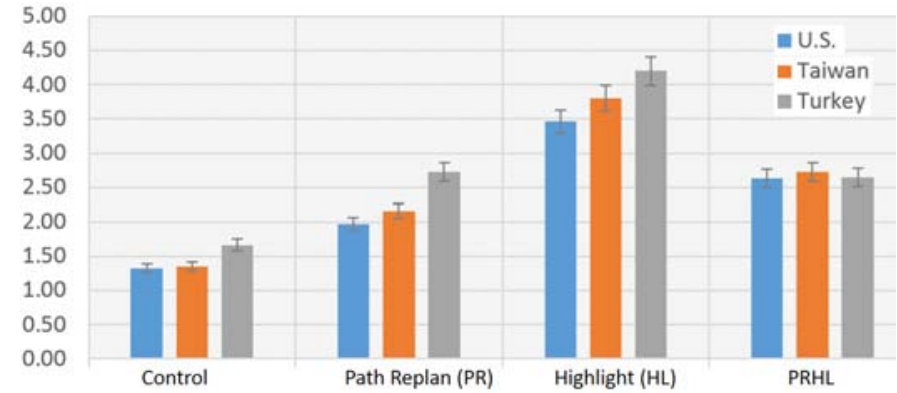

Fig. 8. Vehicle to hazard damage between conditions

\section{E. Compliance}

An operator's compliance in navigation tasks was determined by the ratio of accepted paths to all paths proposed by the conflict detector. Therefore, the analysis only includes PR and PRHL conditions (Fig. 9). Compliance rates ranged from $79 \%$ for Taiwanese participants in the PRHL condition to $22 \%$ for Turkish participants in the PR condition. Although a strategy of accepting all recommendations would have eliminated V-V damage at the cost of a small number of unnecessary reroutings it was not adopted by any of the groups. The results showed a main effect of taskload $\left(\mathrm{F}_{1,348}=4.558, p=\right.$ $.033), \mathrm{DOA} /$ transparency $\left(\mathrm{F}_{1,348}=32.068, p<.001\right)$, and country $\left(\mathrm{F}_{2,348}=5.298, p=.005\right)$, in which higher compliance was observed in the HW conditions as well as in the PRHL condition, confirming TH 1, participants will comply with transparent automation at higher rates. Post-hoc analysis showed that the Taiwanese participants had the highest compliance with the automated recommendations (TW $>\mathrm{US}, \mathrm{p}$ $=.030 ; \mathrm{TW}>\mathrm{TK}, \mathrm{p}=.002$ ), but no difference was observed between the U.S. and the Turkish participants.

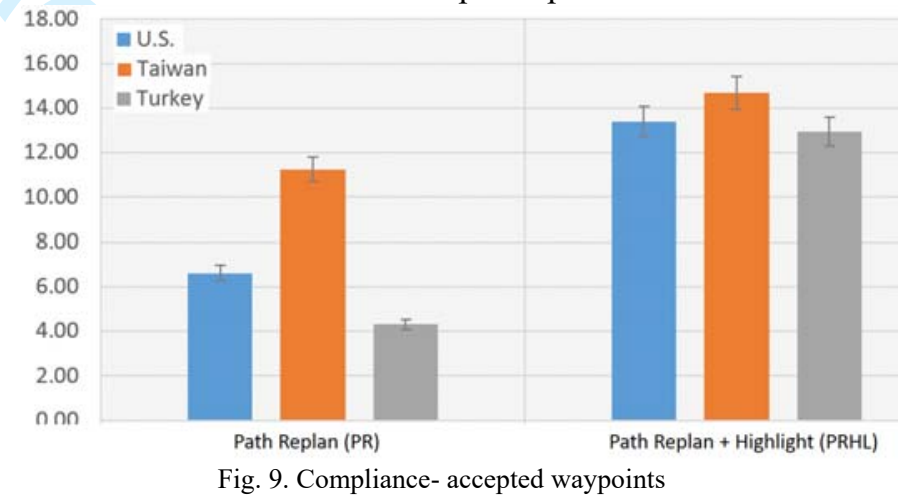

Further analysis showed a cultural effect in the PR condition ( $F 2,174=7.924, p=.001)$ but not on the PRHL condition, in which the Taiwanese participants accepted significantly more proposed new paths in the PR condition than the U.S. participants $(p=.010)$ or the Turkish participants $(p<.001)$. In addition, the number of manual waypoints revealed that American participants added significantly more waypoints (did not comply) than TW ( $p=.036)$ participants in PR condition, and issued a higher number of manual waypoints than TW $(p=$ $.003)$ as well as TK $(p<.001)$ participants in the PRHL condition.

The results (Table II) showed significant correlation with trust in the PR condition where operators could not observe the basis for the automation's decision, but not in the PRHL condition where the conflict being avoided was marked 
TABLE II

CORRELATIONS BETWEEN TRUST AND COMPLIANCE WITH PLANNER Number of Accepted Automated Waypoints in Conflict Detector (Compliance)

\begin{tabular}{|c|c|c|}
\hline \multirow{2}{*}{ Path Re-plan (PR) } & $\begin{array}{c}\text { Pearson } \\
\text { correlation }\end{array}$ & $.300^{* *}$ \\
\cline { 2 - 3 } & Sig. Difference & $\mathbf{. 0 0 0}$ \\
\hline \multirow{2}{*}{$\begin{array}{c}\text { Path Re-plan + Highlight } \\
\text { (PRHL) }\end{array}$} & $\begin{array}{c}\text { Pearson } \\
\text { correlation }\end{array}$ & .092 \\
\cline { 2 - 3 } & Sig. Difference & .218 \\
\hline
\end{tabular}

TABLE III

CORRELATIONS BETWEEN TRUST AND COMPLIANCE BY CULTURE

\begin{tabular}{|c|c|c|}
\hline \multicolumn{2}{|c|}{ Correlation Results } & Path Planner \\
\hline \multirow{2}{*}{$\begin{array}{c}\text { Trust } \\
\underline{U S} \text { group }\end{array}$} & Pearson Correlation & $.274^{*}$ \\
\hline & Sig. (2-tailed) & .034 \\
\hline \multirow{2}{*}{$\begin{array}{c}\text { Trust } \\
\underline{T W} \text { group }\end{array}$} & Pearson Correlation & .171 \\
\hline & Sig. (2-tailed) & .193 \\
\hline \multirow{2}{*}{$\begin{array}{c}\text { Trust } \\
\underline{T K} \text { group }\end{array}$} & Pearson Correlation & $.442 * *$ \\
\hline & Sig. (2-tailed) & .000 \\
\hline
\end{tabular}

allowing operators to base their decision on their observations as well as trust in automation. These observations confirm TH 2 , trust will contribute to dependence under uncertainty and $\mathrm{TH}$ 3 , the role of trust in determining dependence will decrease with decrease in uncertainty. As shown in Table III, trust was a strong determinant of PR use in the Turkish sample and a moderate influence on US participants, but had no effect within the Taiwanese group.

\section{DISCUSSION}

\section{A. Relation Between Trust and Automation Dependence}

In this experiment, trust in automation appears to have served as an effective intervening variable only for the opaque high DOA PR condition as predicted by the unified framework. Its effects on compliance also varied widely across cultures accounting for approximately $20 \%$ of variance in the Turkish sample, $7 \%$ in the US, and none in the Taiwanese. Compliance with the path planner (PR condition) where little other information was available was correlated with trust $(\mathrm{r}=.30)$ and paths were accepted only an average of 5.47 times in the US and Turkish groups. Trust became uncorrelated when highlighting of conflicts was added, yet compliance more than doubled to 13.18 accepted paths, confirming TH 3 , dependence will increase with increasing transparency. In this case the decision to rely on the conflict detector in the PR condition where situation assessment information was unavailable was influenced by trust but when operators were allowed to "see for themselves" (HL and PRHL conditions) trust, although higher, was no longer a necessary basis for usage. Taiwanese participants, by contrast, relied an average of 11.25 times in the opaque PR condition only slightly below their average of 14.67 paths in the PRHL condition. They, however, trusted more in the PRHL condition with ratings in the two conditions (PR and PRHL) comparable to those of the American sample. Only the Turkish sample differentiated trust between the HL and PRHL conditions indicating a minor effect of DOA not resolved by transparency at earlier stages of automation. This difference, however, was dwarfed by those found between the PR and PRHL conditions. These results support the framework's premises of the importance of end-to-end transparency (HL, PRHL) for automation dependence and the relation between automation uncertainty and the role of trust.

\section{B. Transparency and Stages of Automation}

Transparency at earlier (SA) stages provides evidence of the 'cause' of subsequent automated or manual actions. Transparency at the decision and action stage, by contrast, provides only increased precision about outcomes such as more accurate knowledge of results or confidence ratings. Lombrozo [31] argues that humanly intelligible "explanations typically appeal to causes... When explanations are judged for quality the presence of a general pattern is typically preferred to probability judgements alone." Extended to transparency this human preference for teleology should favor choosing transparency at the situation assessment stage where the conditions initiating the automation's actions are observed over transparency at the decision and action stage where only results can be viewed in greater detail. Participants in the PR condition, for example, had clear knowledge of results from observing the conflict avoiding trajectories of the planner and the effects of these actions on the display of running damage providing transparency of performance and evidence of high reliability. They were, however deterred from use, due to uncertainty over why they were being asked to allow the automation to replan paths. When that reassurance was supplied through highlighting, usage tripled within the Turkish sample. Ideally as in [10] and the present study, transparency can be increased across all stages

\section{Culture}

Participants responded to manipulations broadly as expected across the three cultures. Cultural differences for DOA/transparency were found on all four dependent measures. The performance of U.S. and Taiwanese participants was frequently very similar while both differed strongly from the Turkish group. The exception was in response to the opaque PR (path replan) condition in which Taiwanese participants complied with requests at a much higher rate than did their Turkish or American counterparts.

Our cultural predictions, based primarily on the theory of Cultural Syndromes, were confirmed. As hypothesized (H1), dignity culture participants (US) had a higher level of dispositional trust whereas those from the honor culture (Turkey) had a lower level of dispositional trust. Participants from the face culture Taiwan) who were not predicted to be 
either high or low fell in between.

The path re-plan (PR) condition which automated decision and action (DOA) and lacked transparency (did not convey logic of action), had the lowest trust scores confirming H3, Lack of transparency will lower ratings of trust of operators from all cultures and also led to lower compliance for US and TK participants (H4). The highlight (HL) aid, which highlighted possible collisions and hazard violations on the map supporting SA but requiring operators to manually add waypoints, was rated highest in trust. The higher DOA integrated aid which supplied automated path re-planning along with highlighting (PRHL) had an intermediate level of rated trust (due to lower Turkish ratings) but led to substantially higher levels of compliance than either of the other aids.

The shared control within the HL condition was especially beneficial to Turkish participants because honor cultures start with lower levels of trust and may require greater exposure to develop it. While the American and Taiwanese participants had similar levels of trust in the PR and PRHL conditions, which were higher than those of the Turkish participants, no difference was observed in the HL conditions across the three cultures. These findings confirm: H2 Honor culture operators will be less likely to trust high DOA automation than Face or Dignity culture operators.

A higher rate of compliance with the conflict detector (i.e., accepted more proposed new paths) was observed in the PRHL, than in the PR condition. Results show that the American and Turkish participants had both lower trust (H3) and were less likely to comply with automation when situation assessment was unavailable (H4). Taiwanese PR operators also had lower trust scores similar to the Americans however, they continued to rely on opaque automation at a high rate confirming H4. Face culture operators will exhibit automation bias by accepting recommendations from automation even if their basis is not well understood due to their authoritative source, while Dignity and Honor culture operators will be less likely to trust or accept recommendations on this basis.

\section{Counterintuitive V-H Damage Results}

The hypotheses about the interrelated effects of trust, transparency, and DOA were confirmed except for those involving Vehicle-to-Hazard damage which had been predicted to mirror $\mathrm{V}-\mathrm{V}$ damages.

\section{1) V-H Damage Favoring Non Highlight Conditions}

We believe that these counterintuitive findings of higher V$\mathrm{H}$ damage under highlighting conditions (HL, PRHL) and under low taskload resulted from interrelated causes: Ineffectiveness of highlighting of hazard conflicts (HL, PRHL conditions), Greater unaided conspicuity of hazard conflicts than vehicle conflicts (Cntl, PR conditions), Asymmetric allocation of resources, Competition for attention between multiple conflicts.

Hazard violations were indicated in HL and PRHL conditions by difficult to discern dots at points of projected entry and exit of the hazard region as shown in Figure $3 \mathrm{~b}$. We believe this enhancement was ineffective in attracting attention beyond that already provided by the view of the projected path crossing the hazard area. As a consequence, the conspicuity of $\mathrm{V}-\mathrm{H}$ conflicts was effectively the same across the four conditions. The comparison between conditions therefore involves differences in the display of $\mathrm{V}-\mathrm{V}$ conflicts and how they influenced responses to $\mathrm{V}-\mathrm{H}$ conflicts. In HL and PRHL both the $\mathrm{V}-\mathrm{V}$ and $\mathrm{V}-\mathrm{H}$ conflicts are perceptually salient so participants can scan the display to identify the conflict. In the Cntl and PR conditions the V-H conflicts remain perceptually salient but $\mathrm{V}-\mathrm{V}$ conflicts require estimating velocities of the involved vehicles and deciding whether or not they will come too close in the vicinity where their projected paths cross. As a consequence, $\mathrm{Cntl}$ and PR operators for which $\mathrm{V}-\mathrm{H}$ is the only perceptually salient conflict resolve V-H conflicts rapidly accumulating minimal damage, while performance on the estimation based V-V conflicts is much poorer. For HL and PRHL operators both conflicts are perceptually salient leading to potential competitions in which $\mathrm{V}-\mathrm{H}$ conflicts are neglected while more damaging $\mathrm{V}-\mathrm{V}$ ones are resolved.

\section{2) Task Load}

Increasing task load demanded more cognitive resources and required the operators to allocate more attention to the navigation task. Despite frequent findings of reduced trust under higher task loads [16] [21] no effects were observed in the present study, a finding also reported by [22]. There were, however, effects of increased use of automation, often observed when operators must use automation to keep up with task demands [16][21]. Increased load also led to increases in V-V damage. V-H damage, however, was higher in LW, an effect we attribute to the lower rates of compliance and hence increased manual maneuvering for low task load in the aided conditions.

\section{CONCLUSION}

In this study effects of transparency were strongly influenced by culture suggesting that designs and training developed for Western (dignity) cultures may require modification and adjustment for use elsewhere. The distrust leading to disuse found for the Turkish (honor culture) sample suggests that in transitioning automation technologies to honor cultures it may be important to provide extra mechanisms, such as increased transparency, to foster usage. Other features such as providing reassurance through redundant manual controls and extended training periods may be necessary to overcome initial higher levels of distrust. It might be unwise, for example, to introduce driverless cars without a steering wheel, something being planned by two prominent US driverless car firms [37], into honor cultures. The Taiwanese (face culture) showed the opposite tendency toward automation bias in relying on automation in the PR condition even under conditions of reduced trust.

While developed for anthropology the fruitfulness of the theory of Cultural Syndromes for study of human-machine systems was apparent from this study. It readily generated hypotheses, later confirmed, about cultural differences in trust and compliance with automation. Although this study was 
restricted to highly reliable, miss-free automation it is easy to imagine how cultural syndromes might be used to generate hypotheses about automation usage decisions of unreliable automation conditions as well as in other areas of humanmachine studies.

\section{REFERENCES}

[1] R. House, P. Hanges, and M. Javidan, Culture, leadership, and organizations. Thousand Oaks: SAGE Publications, 2004.

[2] Hofstede, Cultures And Organizations - Software of the Mind. New York: McGraw-Hill., 1991.

[3] S. Aslani, J. Ramirez-Marin, J. Brett, J. Yao, Z. Semnani-Azad, Z.X. Zhang, C. Tinsley, L. Weingart, and W. Adair, "Dignity, face, and honor cultures: A study of negotiation strategy and outcomes in three cultures," J. Organ. Behav., vol. 37, no. 8, pp. 1178-1201, Nov. 2016.

[4] B. Yoo, N. Donthu, and T. Lenartowicz, "Measuring Hofstede' s Five Dimensions of Cultural Values at the Individual Level : Development and Validation of CVSCALE Measuring Hofstede' s Five Dimensions of Cultural Values at the Individual Level," J. Int. Consum. Mark., vol. 23, no. August 2011, pp. 193-210, 2011.

[5] A. K. Y. Leung and D. Cohen, "Within-and between-culture variation: Individual differences and the cultural logics of honor, face, and dignity cultures," J. Pers. Soc. Psychol., vol. 100, no. 3, pp. 507-526, 2011.

[6] T. Yamagishi, K. Cook, and M. Watabe, "Uncertainty, Trust, and Commitment Formation in the United States and Japan 1," Am. J. Sociol., vol. 104, no. 1, pp. 165-194, 1998.

[7] J. Nisbett and D. Cohen, "Culture of Honor The Psychology of Violence in the South," South. Cult., vol. 3, no. 1, pp. 80-83, 1997.

[8] H. P. Beck, M. T. Dzindolet, and L. G. Pierce, "Automation Usage Decisions: Controlling Intent and Appraisal Errors in a Target Detection Task," Hum. Factors J. Hum. Factors Ergon. Soc., vol. 49, no. 3, pp. 429-437, 2007.

[9] K. A. Hoff and M. Bashir, "Trust in Automation: Integrating Empirical Evidence on Factors That Influence Trust ," Hum. Factors J. Hum. Factors Ergon. Soc., vol. 57, no. 3, pp. 407-434, 2015.

[10] J. B. Lyons, K. S. Koltai, N. T. Ho, W. B. Johnson, D. E. Smith, and R. J. Shively, "Engineering Trust in Complex Automated Systems," Ergon. Des. Q. Hum. Factors Appl., vol. 24, no. 1, pp. 13-17, Jan. 2016.

[11] N. Wang, D. V. Pynadath, and S. G. Hill, "Trust calibration within a human-robot team: Comparing automatically generated explanations," ACM/IEEE Int. Conf. Human-Robot Interact., vol. 2016-April, pp. 109-116, 2016.

[12] J. D. Lee and K. A. See, "Trust in Automation: Designing for Appropriate Reliance," Hum. Factors J. Hum. Factors Ergon. Soc., vol. 46, no. 1, pp. 50-80, 2004.

[13] K. E. Schaefer, J. Y. C. Chen, J. L. Szalma, and P. A. Hancock, “A Meta-Analysis of Factors Influencing the Development of Trust in Automation," Hum. Factors J. Hum. Factors Ergon. Soc., vol. 58, no. 3, pp. 377-400, May 2016.

[14] R. Wiczorek and D. Manzey, "Is Operators' Compliance with Alarm Systems a Product of Rational Consideration?," in Proceedings of the Human Factors and Ergonomics Society Annual Meeting, 2010, pp. 1722-1726.

[15] E. T. Chancey, J. P. Bliss, Y. Yamani, and H. A. H. Handley, "Trust and the Compliance-Reliance Paradigm: The Effects of Risk, Error Bias, and Reliability on Trust and Dependence," Hum. Factors, vol. 59, no. 3, pp. 333-345, 2017.

[16] E. Rovira, K. McGarry, and R. Parasuraman, "Effects of Imperfect Automation on Decision Making in a Simulated Command and Control Task," Hum. Factors J. Hum. Factors Ergon. Soc., vol. 49, no. 1, pp. 76-87, Feb. 2007.

[17] F. M. F. Verberne, J. Ham, and C. J. H. Midden, "Trust in Smart Systems: Sharing Driving Goals and Giving Information to Increase Trustworthiness and Acceptability of Smart Systems in Cars," no. 1995, 2004.

[18] J. Y. C. Chen, K. Procci, M. Boyce, J. Wright, A. Garcia, and M. J. Barnes, "Situation Awareness - Based Agent Transparency," 2014.
[19] J. D. Lee and N. Moray, "Trust, self-confidence, and operators' adaptation to automation," Int. J. Hum. Comput. Stud., vol. 40, no. 1, pp. 153-184, 1994.

[20] L. Wang, P.-L. P. Rau, V. Evers, B. K. Robinson, and P. Hinds, "When in Rome: the role of culture \& context in adherence to robot recommendations," in Proceeding of the 5th ACM/IEEE international conference on Human-robot interaction - HRI '10, 2010, pp. 359-366.

[21] D. P. Biros, M. Daly, and G. Gunsch, "The influence of task load and automation trust on deception detection," Gr. Decis. Negot., vol. 13, no. 2, pp. 173-189, 2004.

[22] B. Rajaonah, N. Tricot, F. Anceaux, and P. Millot, "The role of intervening variables in driver-ACC cooperation," Int. J. Hum. Comput. Stud., vol. 66, no. 3, pp. 185-197, 2008.

[23] R. Parasuraman, T. B. Sheridan, and C. D. Wickens, "A model for types and levels of human interaction with automation.," IEEE Trans. Syst. Man. Cybern. A Syst. Hum., vol. 30, no. 3, pp. 286-97, May 2000.

[24] L. Onnasch, C. D. Wickens, H. Li, and D. Manzey, "Human performance consequences of stages and levels of automation: An integrated meta-analysis," Hum. Factors, vol. 56, no. 3, pp. 476488, 2014.

[25] D. B. Kaber and M. R. Endsley, "Out-of-the-loop performance problems and the use of intermediate levels of automation for improved control system functioning and safety," Process Saf. Prog., vol. 16, no. 3, pp. 126-131, 1997.

[26] G. Calhoun, M. Draper, and H. Ruff, "Effect of level of automation on unmanned aerial vehicle routing task," Proc. Hum. ..., pp. 197201, 2009.

[27] F. Amato, M. Felici, P. Lanzi, G. Lotti, L. Save, and A. Tedeschi, "Trust Observations in Validation Exercises," in 2011 Fifth International Conference on Secure Software Integration and Reliability Improvement, 2011, pp. 216-223.

[28] N. Dadashi, A. W. Stedmon, and T. P. Pridmore, "Semi-automated CCTV surveillance: The effects of system confidence, system accuracy and task complexity on operator vigilance, reliance and workload," Appl. Ergon., vol. 44, no. 5, pp. 730-738, 2013.

[29] P. Wright, Julia, Chen, Jessie, Barnes, Michael, Hancock, "The Effect of Agent Reasoning Transparency on Automation Bias: An Analysis of Response Performance," in LNCS Virtual, Augmented, and Mixed Reality, 2016, pp. 465-477.

[30] G. Sadler, H. Battiste, N. Ho, L. Hoffmann, W. Johnson, R. Shively, J. Lyons, and D. Smith, "Effects of transparency on pilot trust and agreement in the autonomous constrained flight planner," AIAA/IEEE Digit. Avion. Syst. Conf. - Proc., vol. 2016-Decem, pp. $1-9,2016$.

[31] T. Lombrozo, "The structure and function of explanations," Trends Cogn. Sci., vol. 10, no. 10, pp. 464-470, 2006.

[32] Y. Boussemart and M. Cummings, "Behavioral recognition and prediction of an operator supervising multiple heterogeneous unmanned vehicles," in Humans Operating Unmanned Systems, 2008, pp. 1-12.

[33] T. Johns, "On Curves of Minimal Length with a Constraint on Average Curvature, and with Prescribed Initial and Terminal Positions and Tangents Author (s ): L . E . Dubins Source: American Journal of Mathematics, Vol . 79 , No . 3 ( Jul ., 1957), pp . 497-516 Pu," vol. 3, no. 3, pp. 497-516, 2016.

[34] S.-Y. Chien, M. Lewis, K. Sycara, J.-S. Liu, and A. Kumru, "OpenICPSR data archive: The Influence of Cultural Factors on Trust in Automation," 2017. [Online]. Available: http://doi.org/10.3886/E100532V3. [Accessed: 14-May-2018].

[35] A. Chien, Shih-yi, Lewis, Michael, Sycara, Katia, Liu, Jyi-Shane, Kumru, "The Effect of Culture on Trust in Automation: Reliability and Workload," ACM Trans. Interact. Intell. Syst.

[36] S. G. Hart and L. E. Staveland, "Development of NASA-TLX (Task Load Index): Results of Empirical and Theoretical Research," in Human Mental Workload, vol. 1, North-Holland, 1988, pp. 139183.

[37] NPR, "gm-says-car-with-no-steering-wheel-or-pedals-ready-forstreets," NPR.org, 2018. [Online]. Available: https://www.npr.org/sections/thetwoway/2018/01/12/577688125/gm-says-car-with-no-steering-wheelor-pedals-ready-for-streets-in-2019. [Accessed: 10-Oct-2018]. 


\begin{tabular}{|c|c|c|c|c|c|c|}
\hline \multicolumn{7}{|c|}{$\begin{array}{l}\text { General Trust Scale } \\
\text { (administered before use) }\end{array}$} \\
\hline Dimension & Survey Items & $\begin{array}{l}\text { Disagree } \\
\text { strongly }\end{array}$ & Disagree & $\begin{array}{l}\text { Neither } \\
\text { agree nor } \\
\text { disagree }\end{array}$ & Agree & $\begin{array}{l}\text { Agree } \\
\text { strongly }\end{array}$ \\
\hline \multirow{3}{*}{$\begin{array}{l}\text { General } \\
\text { Automation } \\
\text { Performance } \\
\text { Expectancy }\end{array}$} & $\begin{array}{l}\text { Using a decision aid will } \\
\text { increase my effectiveness on my } \\
\text { jobs. }\end{array}$ & 1 & 2 & 3 & 4 & 5 \\
\hline & $\begin{array}{l}\text { Using decision aids will } \\
\text { improve my output quality. }\end{array}$ & 1 & 2 & 3 & 4 & 5 \\
\hline & $\begin{array}{l}\text { Using a decision aid will } \\
\text { increase my chances of } \\
\text { achieving a higher level of } \\
\text { performance. }\end{array}$ & 1 & 2 & 3 & 4 & 5 \\
\hline \multirow{3}{*}{$\begin{array}{l}\text { General } \\
\text { Automation } \\
\text { Process } \\
\text { Transparency }\end{array}$} & $\begin{array}{l}\text { The information that a decision } \\
\text { aid provides is of high quality. }\end{array}$ & 1 & 2 & 3 & 4 & 5 \\
\hline & $\begin{array}{l}\text { A decision aid provides } \\
\text { sufficient information. }\end{array}$ & 1 & 2 & 3 & 4 & 5 \\
\hline & $\begin{array}{l}\text { I am satisfied with the } \\
\text { information that a decision aid } \\
\text { provides. }\end{array}$ & 1 & 2 & 3 & 4 & 5 \\
\hline \multirow{3}{*}{$\begin{array}{l}\text { General } \\
\text { Automation } \\
\text { Cultural- } \\
\text { Technological } \\
\text { Context }\end{array}$} & $\begin{array}{l}\text { I prefer to use a decision aid to } \\
\text { make decisions under high } \\
\text { workload situations. }\end{array}$ & 1 & 2 & 3 & 4 & 5 \\
\hline & $\begin{array}{l}\text { Using a decision aid helps me to } \\
\text { expend less effort to accomplish } \\
\text { tasks. }\end{array}$ & 1 & 2 & 3 & 4 & 5 \\
\hline & $\begin{array}{l}\text { Using a decision aid helps me } \\
\text { accomplish tasks with lower } \\
\text { risk. }\end{array}$ & 1 & 2 & 3 & 4 & 5 \\
\hline \multicolumn{7}{|c|}{$\begin{array}{l}\text { Specific Trust Scale } \\
\text { (administered following use) }\end{array}$} \\
\hline \multirow{3}{*}{$\begin{array}{l}\text { Specific } \\
\text { Automation } \\
\text { Performance } \\
\text { Expectancy }\end{array}$} & $\begin{array}{l}\text { The conflict detector improves } \\
\text { my performance. }\end{array}$ & 1 & 2 & 3 & 4 & 5 \\
\hline & $\begin{array}{l}\text { The conflict detector enables } \\
\text { me to accomplish tasks more } \\
\text { quickly. }\end{array}$ & 1 & 2 & 3 & 4 & 5 \\
\hline & $\begin{array}{l}\text { The conflict detector increases } \\
\text { my productivity. }\end{array}$ & 1 & 2 & 3 & 4 & 5 \\
\hline \multirow{3}{*}{$\begin{array}{l}\text { Specific } \\
\text { Automation } \\
\text { Process } \\
\text { Transparency }\end{array}$} & $\begin{array}{l}\text { My interaction with the conflict } \\
\text { detector is clearly } \\
\text { understandable. }\end{array}$ & 1 & 2 & 3 & 4 & 5 \\
\hline & $\begin{array}{l}\text { The conflict detector is user- } \\
\text { friendly. }\end{array}$ & 1 & 2 & 3 & 4 & 5 \\
\hline & $\begin{array}{l}\text { The conflict detector uses } \\
\text { appropriate methods to reach } \\
\text { decisions. }\end{array}$ & 1 & 2 & 3 & 4 & 5 \\
\hline \multirow{3}{*}{$\begin{array}{l}\text { Specific } \\
\text { Automation } \\
\text { Purpose } \\
\text { Influence }\end{array}$} & $\begin{array}{l}\text { I am confident about the } \\
\text { performance of the conflict } \\
\text { detector. }\end{array}$ & 1 & 2 & 3 & 4 & 5 \\
\hline & $\begin{array}{l}\text { When an emergent issue or } \\
\text { problem arises, I would feel } \\
\text { comfortable depending on the } \\
\text { information provided the } \\
\text { conflict detector. }\end{array}$ & 1 & 2 & 3 & 4 & 5 \\
\hline & $\begin{array}{l}\text { I can always rely on the conflict } \\
\text { detector to ensure my } \\
\text { performance. }\end{array}$ & 1 & 2 & 3 & 4 & 5 \\
\hline
\end{tabular}

